\title{
Zipping it up: DEAs independent of the elastomer's electric breakdown field
}

\author{
Pit Gebbers*a,b, Chauncey Grätzel ${ }^{\mathrm{c}}$, Luc Maffli ${ }^{\mathrm{a}}$, Christoph Stamm ${ }^{\mathrm{b}}$, Herbert Shea ${ }^{\mathrm{a}}$ \\ ${ }^{a}$ EPFL-LMTS, Jaquet-Droz 1, CP 526, 2002 Neuchâtel, Switzerland; \\ bZHAW-ZAMP, Technikumstrasse 9, 8401 Winterthur, Switzerland; \\ 'Optotune AG, Bernstrasse 388, 8953 Dietikon, Switzerland
}

\begin{abstract}
We demonstrate here an alternative dielectric elastomer actuator (DEA) structure, which relies on the compliant nature of elastomer membranes but does not require any electric field in the elastomer. Our elastomer zipping device is a macroscopic version of the electrostatic zipping actuators common in silicon MEMS. It consists of a cm-sized metallic bottom electrode, covered by a thin insulator, on which the elastomer membrane is bonded, enclosing a tapered air gap. A compliant electrode is patterned on the lower face of the elastomer membrane. Applying a voltage between solid bottom electrode and compliant electrode leads to controlled pull-in in movement, comparable to the closing of a zipper, thus giving large strokes and forces with no electrical requirements on the elastomer since no voltage is applied across the membrane. The compliant electrodes $(20 \mathrm{~mm}$ diameter $)$ are produced by metal ion-implantation into the elastomer membranes. The bottom metal electrodes are coated with 10 to $30 \mu \mathrm{m}$ of $\mathrm{Al}_{2} \mathrm{O}_{3}$. We report on our experimental study of membrane deflection and dynamics and discuss the effect of design parameters such as elastomer mechanical properties and actuator geometry. Membrane deflection of up to $1.4 \mathrm{~mm}$ was reached at only $200 \mathrm{~V}$ actuation voltage. The large membrane deformation achieved with this zipping actuation can be applied to applications such as pumps or tunable liquid lenses. The out-of plane movement of the membrane can be used for linear actuation.
\end{abstract}

Keywords: DEA, electrostatic zipping actuator

\section{INTRODUCTION}

\subsection{Dielectric Elastomer Actuators}

Dielectric Elastomer Actuators (DEAs) represent a popular type of electroactive polymers ${ }^{1-3}$. They consist of an elastomer membrane, covered on both sides with compliant electrodes. When a voltage is applied to the electrodes, electrostatic forces act on the membrane, causing it to be compressed. The resulting pressure $\mathrm{P}$ is given by

$$
P=\varepsilon_{0} \varepsilon_{r} E^{2}
$$

with $E$ as electric field across the membrane, $\varepsilon_{0}$ is the vacuum permittivity and $\varepsilon_{\mathrm{r}}$ as the relative dielectric constant of the elastomer.

In many cases, the maximal pressure is what limits the actuator performance. Equation (1) shows that $P$ can be increased in two ways: by choosing a material with a high dielectric constant, or by increasing the electric field across the elastomer. However, these two values are linked: for any polymer, polar groups increase the dielectric constant, and at the same time increase ionic conductance, along with lowering the mobility of the molecular chains ${ }^{3}$. Choosing an elastomer with high $\varepsilon_{\mathrm{r}}$ will consequently often result in lower dielectric strength of the membrane. This limits the maximal attainable pressure. Additionally, the lower molecular chain mobility will make the material stiffer, reducing the strain of the material. This limits the displacement reached by the actuator.

Decoupling mechanical from electrical actuator properties would therefore allow more freedom in the actuator design. We present here an alternative actuator structure, which is not limited by these coupling constraints.

\footnotetext{
* Send correspondence to Pit Gebbers: pit.gebbers@gmail.com
} 


\subsection{Electrostatic zipping actuators in MEMS}

In MEMS, electrostatic actuation is widely used ${ }^{4,5}$. The most frequent geometries are the parallel plate and the combdrive structures. In parallel plate actuators the force between the two plates is given by

$$
F=\frac{\varepsilon A V^{2}}{2 x^{2}}
$$

with $\varepsilon$ being the permittivity, $A$ the plate area, $V$ the applied voltage, and $x$ the electrode distance.

In comb drive actuators, both electrodes are fabricated in comb shape, and assembled so that the teeth of the two structures are interdigitated. In this configuration, the attractive force is a linear function of the distance:

$$
F=\frac{n t \varepsilon V^{2}}{g}
$$

with $n$ the number of teeth, $t$ the thickness of the electrode, and $g$ the gap between the electrodes.

As an alternative to those widely-used structures, the zipping actuator has been proposed and studied ${ }^{6-8}$. Unlike the parallel plate types, where pull-in is an undesired and avoided side effect, zipping actuators operate exclusively in a regime where the electrodes are in partial pull-in.

In an electrostatic zipping actuator, at least one of the electrodes is mechanically flexible. One electrode, typically the rigid one, is covered with a thin insulator layer. The electrodes are arranged in an angle to each other, making contact at one end. When a voltage is applied, the electrostatic forces are highest where the electrodes touch, locally deforming the compliant electrode and pressing it onto the rigid electrode (Figure 1). As the voltage increases, this effect progresses, leading to a motion similar to a zipper.

As zipping actuator topology makes use of the pull-in effect, it is not limited by it and allows large displacements.

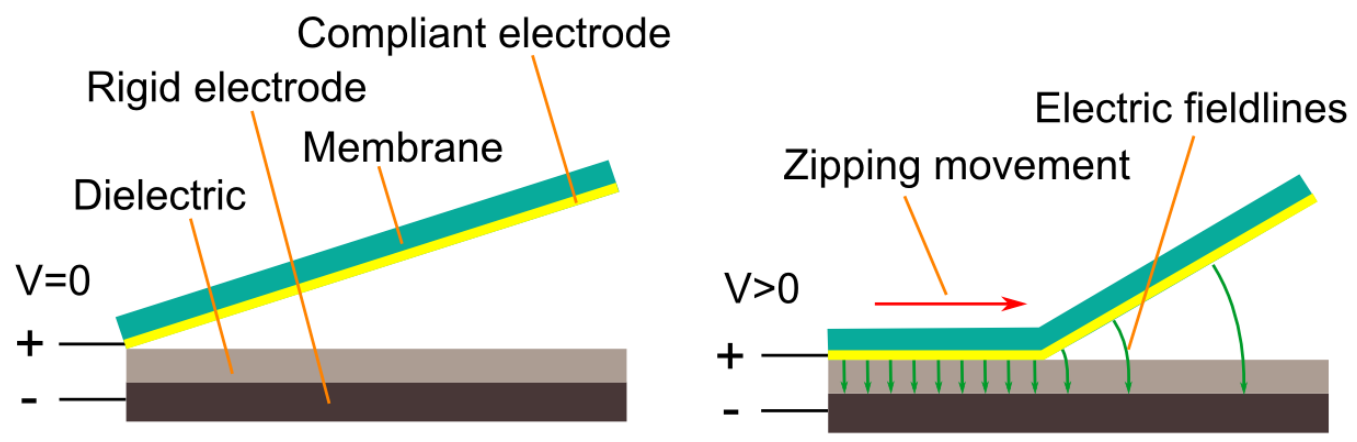

Figure 1: Schematic zipping actuator. The top element represents the compliant electrode, the bottom element the insulator layer and the rigid electrode. The red lines on the right drawing symbolize the electric field.

\subsection{Elastomer zipping actuators}

In addition to their use in MEMS, zipping actuators have also been proposed for macroscopic applications, especially for membrane pumps ${ }^{9-12}$. The membranes used in such pumps are typically made of metal or similarly rigid materials.

The concept of zipping actuation has also been applied to polymers ${ }^{13}$, 14 . The low-stiffness polymer material is used as a substrate for the compliant electrode of the actuators. Horning et al. proposed stacked zipping actuators that consist of a number of zipping cells, which in combination produce a high-force, low power actuator ${ }^{13}$. Binh-Khiem et al. created tunable lenses actuated by zipping actuators produced by depositing a parylene layer and a gold electrode onto a drop of liquid ${ }^{14}$.

In this work, we demonstrate macroscopic zipping actuators with the use of soft elastomer membranes. 


\section{EXPERIMENTAL CHARACTERIZATION}

\subsection{Device fabrication}

We have built different types of elastomer zipping actuators, which can be divided in two groups: devices with axisymmetric geometry, and non-symmetric devices.

The axi-symmetric actuator was designed as depicted in Figure 2. It contains a machined metal part that acts as rigid electrode. The electrode has the shape of a bowl with a central hole and is covered with a thin insulating layer. The membrane is glued to a support ring, and covers the bowl opening. One side of the membrane is covered with a compliant electrode and faces the rigid electrode. The rigid electrode and the membrane ring are held by a support structure. When a voltage is applied between the two electrodes, the membrane is pulled down onto the rigid electrode, starting from the outer rim, zipping towards the center. This causes the membrane to perform an out-of-plane movement. With a $20 \mathrm{~mm}$ diameter rigid electrode and a hole of $3 \mathrm{~mm}$ diameter, up to $1 \mathrm{~mm}$ of axial movement is reached. The central hole allows the central region of the membrane to be accessed from both sides, so that the device may be used for application like lens displacement inside a small optical system.
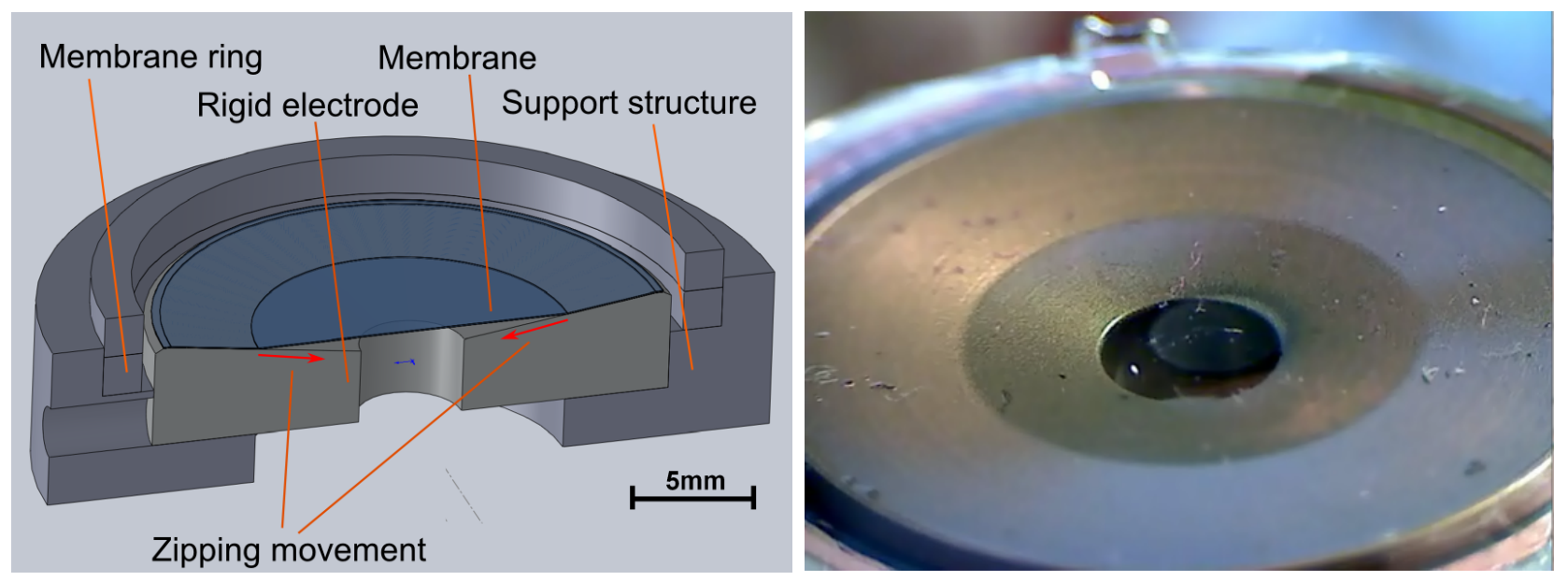

Figure 2: Drawing and photograph of our axi-symmetrical elastomer zipping actuator. With voltage applied, the membrane is pulled down to the electrode, starting from the outer rim of the rigid electrode towards the center. The center of the membrane is displaced in an out-of-plane movement along the axis of symmetry.

The second group of our actuators has a non-symmetric configuration. Two examples are shown in Figure 3. The rigid electrode consists of a metal plate, which is either flat, or deformed to have a specific curvature. The side length of the plates is between 8.5 and $18 \mathrm{~mm}$. They are covered with a thin insulating layer. A ring supports a membrane with a compliant electrode on the bottom side. The rigid electrode is in contact with the membrane in a specific angle and is held in place by means of external fixtures. When a voltage is applied, the membrane is pulled down progressively onto the rigid electrode, beginning at the initial point of contact and moving in the direction of the slope.

Compared to the axi-symmetrical devices, the rigid electrode of the non-symmetrical devices has a simpler structure and allows for easier fabrication at random surface curvatures. All experiments presented in this work were performed with these asymmetric devices. 

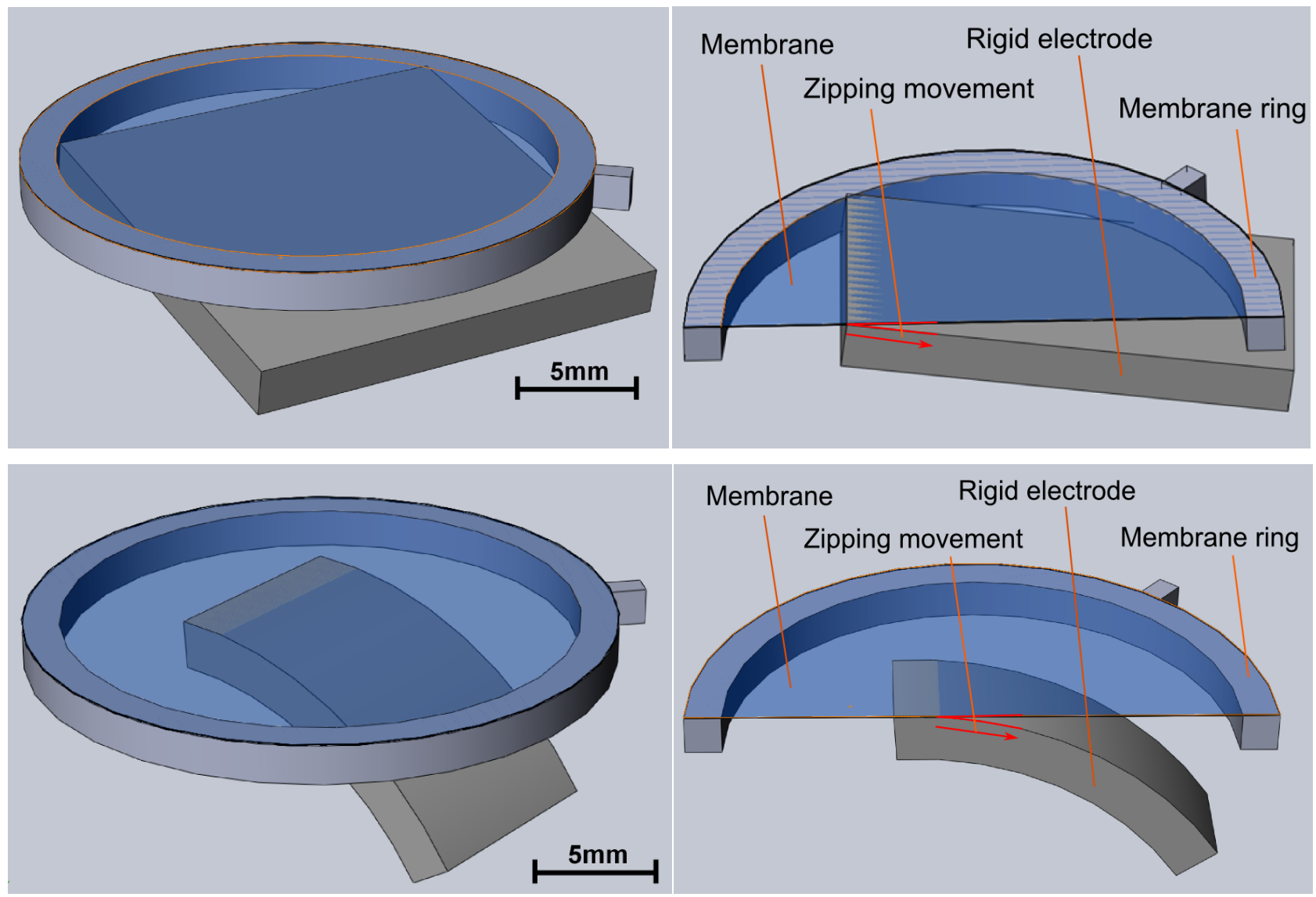

Figure 3: Non-symmetric test actuators. The rigid electrode touches the membrane at specific angles. The elements are held in place by external fixtures (not shown). When voltage is applied, the membrane is progressively pulled down onto the rigid electrode in the direction of the red arrows. These actuator structures were used for the experiments presented in this work. Top: flat version. Bottom: curved version.

\section{Compliant element}

For the compliant element of our zipping actuators, we used membranes made of silicone elastomer. The material's young's modulus is $\mathrm{Y}=0.4 \mathrm{MPa}$. All membranes had a thickness before prestretch of $\mathrm{t}_{0}=50 \mu \mathrm{m}$. The membranes were pre-stretched by different ratios. Table 1 shows the membranes used in the presented test actuators.

To make the membranes work in the zipping actuators, one side of the elastomer needs to be covered with an electrode. This electrode must be compliant and mechanically stable: its adhesion forces to the elastomer material must be higher than the occurring electrostatic forces in order to prevent mechanical failure. As electrostatic forces scales inversely with the square of the thickness of the dielectric between the rigid and fixed electrodes, no additional insulating layers should be formed on top of the flexible electrode. The inter-electrode distance is further minimized if both electrode surfaces have low roughness.

We have found that a well-suited method for creating such electrodes is low-energy metal ion implantation. In this process, metal ions are accelerated to energies around $5 \mathrm{keV}$ and directed onto the target membrane ${ }^{15-18}$. A shadow mask is used to pattern the electrode shape. At those comparatively low energies, the penetration depth of the ions in the elastomer material remains low, typically only a few tens of nanometers ${ }^{19}$. The ions form a thin layer of metal clusters right beneath the surface. As the clusters are in contact with each other, the layer is electrically conductive, but also compliant, as no continuous metal sheet is formed. The surface roughness has been shown to remain low: typical metal ion implantation increased $R_{\text {rms }}$ of a PDMS membrane from $2 \mathrm{~nm}$ to values below $20 \mathrm{~nm}^{18}$. 
The close proximity of the particle sheet to the elastomer surface minimizes the occurrence of a passive layer that would diminish electrostatic forces in a zipping actuator, since the few nm thick passive layer is 3 orders of magnitude thinner than the $\mu \mathrm{m}$ of oxide grown on the rigid electrode.

Another interesting effect is the fact that the thin metal layer hardens the membrane material very locally. As stiction is diminished with increasing material stiffness ${ }^{20}$, this reduces unwanted adhesion between membrane and insulator.

Each membrane used in the presented experiments was first prestretched and glued onto a temporary support ring. Then, the compliant electrode was implanted using our laboratory's filtered cathodic vacuum arc implanter ${ }^{15,16}$. A steel shadow mask was used to pattern the electrode. Finally, the membranes were glued onto an epoxy support ring. A copper track on the ring makes physical contact with the ion implanted membrane and is used to apply the actuation voltage.

Table 1: Properties of the membranes used in the presented experiments.

\begin{tabular}{|l|l|l|l|l|}
\hline $\begin{array}{l}\text { Elastomer } \\
\text { young's } \\
\text { modulus } \\
(\mathrm{MPa})\end{array}$ & $\begin{array}{l}\text { Initial } \\
\text { thickness } \\
(\mu \mathrm{m})\end{array}$ & $\begin{array}{l}\text { Prestretch } \\
(\%)\end{array}$ & $\begin{array}{l}\text { Thickness } \\
\text { after prestretch } \\
(\mu \mathrm{m})\end{array}$ & $\begin{array}{l}\text { Implantation dose } \\
\left(\mathrm{at} / \mathrm{cm}^{2}\right)\end{array}$ \\
\hline \multirow{3}{*}{0.4} & \multirow{2}{*}{50} & 0 & 50 & $1.15 \times 10^{16}$ \\
\cline { 3 - 5 } & 25 & 32 & $0.80 \times 10^{16}$ \\
\cline { 3 - 5 } & 50 & 22.2 & $0.79 \times 10^{16}$ \\
\hline
\end{tabular}

\section{Rigid electrode}

We fabricated the rigid electrode out of the aluminum alloy AlMg1 (Mg $1 \% \mathrm{wt})$. For the test actuators presented here, geometries with different surface shapes were used: some with a flat square surface (17 mm side length), and others with curved surfaces (width $8.5 \mathrm{~mm}$, radii of curvature $7-15 \mathrm{~mm}$ ).

For zipping actuators, the rigid electrode has to be covered with an insulator layer. An ideal insulator for zipping actuation has a high dielectric strength, high dielectric constant, and is mechanically stable at low thickness.

To select the insulator material, we compared the maximal force attainable by specific material parameters. When reviewing the research undertaken by groups studying zipping actuators in MEMS, it can be seen that in the reported formulas, the maximum actuator force for a given insulator thickness is proportional to the factor $\varepsilon_{r}^{3 / 4} E_{b d}{ }_{b / 2}^{3 / 2}$ (with $\varepsilon_{r}$ as the insulator material's relative dielectric constant, and $E_{b d}$ the electric breakdown field of the dielectric) ${ }^{7,8}$. We compared this factor for different insulator materials as shown in Table 2. It can be seen that aluminum oxide clearly shows the highest performance factor. What needs to be considered, however, is that this comparison assumes perfectly homogenous insulator layers, whereas in reality, the fabrication process greatly influences the final performance of the insulator. Still, the data points out that aluminum oxide has a high potential to be used in zipping actuators, and was chosen for the here presented test devices.

Table 2: Comparison of insulator materials regarding their performance in zipping actuators. Material data taken from ${ }^{21}$.

\begin{tabular}{|c|c|c|c|}
\hline Material & $\begin{array}{c}\text { Dielectric } \\
\text { constant } \varepsilon_{\mathrm{r}}\end{array}$ & $\begin{array}{c}\text { Electric breakdown } \\
\text { field } \mathrm{E}_{\mathrm{bd}}(\mathrm{MV} / \mathrm{cm})\end{array}$ & $\varepsilon_{\mathrm{r}}^{3 / 4} \mathrm{E}_{\mathrm{bd}}{ }^{3 / 2}$ \\
\hline $\mathrm{SiO}_{2}$ & 3.9 & 15 & 161.2 \\
\hline $\mathrm{Al}_{2} \mathrm{O}_{3}$ & 9 & 11.2 & 194.8 \\
\hline $\mathrm{HfO}_{2}$ & 25 & 3.9 & 86.1 \\
\hline $\mathrm{Ta}_{2} \mathrm{O}_{5}$ & 26 & 2.6 & 48.3 \\
\hline $\mathrm{TiO}_{2}$ & 95 & 1 & 30.4 \\
\hline $\mathrm{SrTiO}_{3}$ & 200 & 0.8 & 38.1 \\
\hline
\end{tabular}


The $\mathrm{Al}_{2} \mathrm{O}_{3}$ insulator layer was formed by anodizing the part surfaces. Layers of 10-30 $\mu \mathrm{m}$ thickness were grown by a commercial provider of surface anodizing.

In the anodization process, the aluminum surface is oxidized electrochemically. By controlling the electrolyte composition, the applied voltage and the process duration, the properties of the forming alumina film can be controlled. The result is a mechanically very stable and electrically insulating layer. In most anodization processes, however, the resulting layer shows the presence of pores, which reduce its insulating capabilities. In our parts, this is shown by the behavior of the insulator resistance when the elements are heated in the oven (Figure 4): the resistivity drastically increases after 50 minutes at $100^{\circ} \mathrm{C}$, most likely because of the evaporation of moisture trapped in the pores.

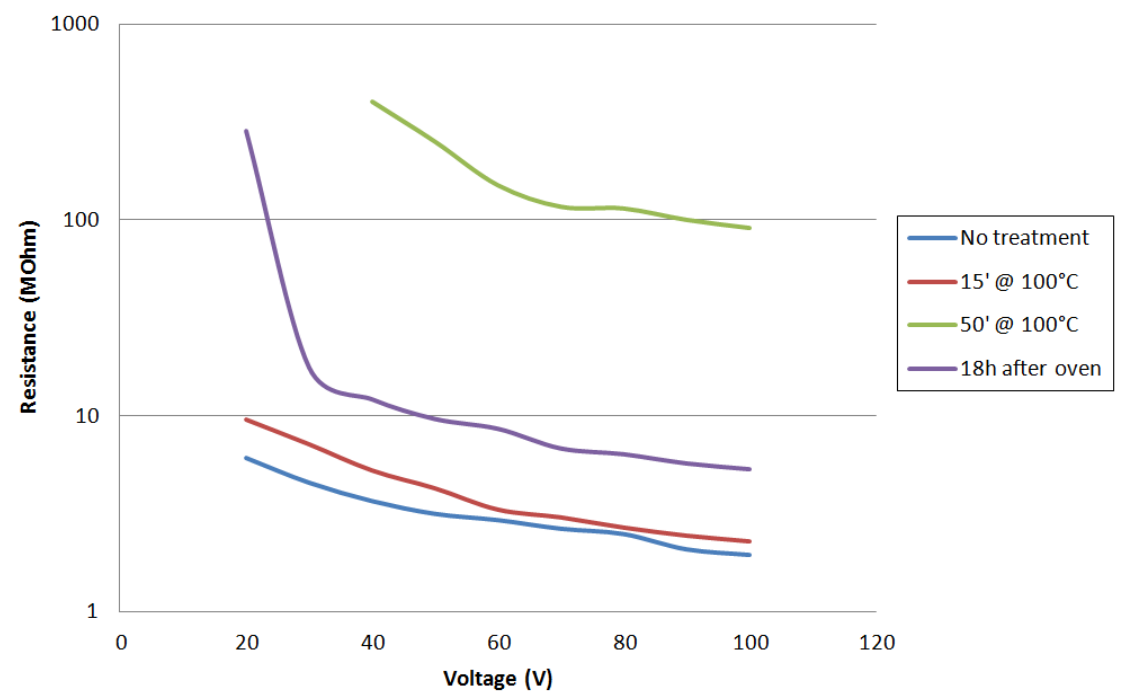

Figure 4: Voltage-dependent insulator resistance of a $300 \mathrm{~mm}^{2} \mathrm{Al}$ surface anodized with a $25 \mu \mathrm{m}$ layer of $\mathrm{Al}_{2} \mathrm{O}_{3}$. Measurements were taken of the same part before and after baking it in an oven at $100^{\circ} \mathrm{C}$ for different periods of time, and after leaving it for 18 hours at RT afterwards.

\section{Actuators with liquid}

There exist several applications where elastomer zipping actuators may be used in combination with a liquid, such as membrane pumps or tunable optical lenses (see section 1.3). To study the effect of liquid on our zipping actuators, we fabricated the device shown in Figure 5. The membrane was produced in the same way as described above and glued to a support ring. Then, a spacer ring was glued on the side on the membrane that had no electrode implanted. A cover glass was glued on top of the spacer. Two holes were drilled in the spacer ring and distilled water was injected into one of the holes using a syringe. During the injection, the second hole allowed the air trapped inside the chamber to escape. The amount of injected liquid was controlled so that the membrane of the resulting device would be flat, i.e. in a way that it would not bulge neither inwards nor outwards. The filling holes were then closed with bolts. 

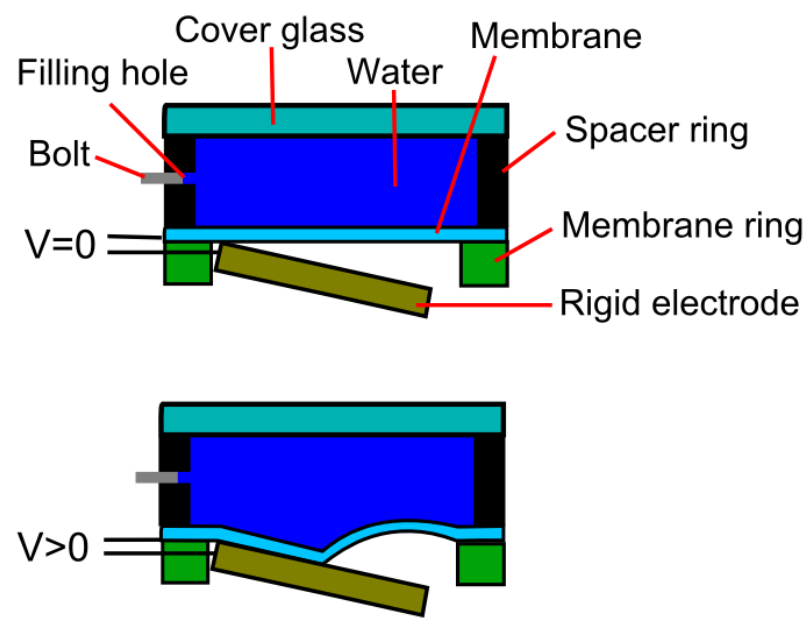

Figure 5: Cross section of our elastomer zipping actuator enclosing a liquid. A spacer ring is glued on top of the membrane, and closed with a cover glass. The resulting space is filled with distilled water. The compliant electrode implanted in the membrane faces the side of the rigid electrode.

\subsection{Experimental setup}

For actuator characterization, we used a camera based test setup as shown in Figure 6. The assembled device is placed beneath a camera, and the response of the actuator is captured by still images or video. A USB microscope (dnt DigiMicro 2.0) was use to capture still images of static displacement measurements. For the dynamic experiments, a compact camera with good macro capability (Olympus VR-310) was used to record videos at 30fps. A printed scale placed beside the device serves as a reference to relate the image distance to real-world dimensions. Figure 7 shows two sample images of an actuator with and without voltage applied.

The captured image data is then analyzed using a software tool (ImageJ with manual tracking plugin). The tool is used to track the lateral displacement of the point of contact between membrane and electrode ( $\left.\mathrm{x}_{\text {meas }}\right)$. For this, a virtual line is drawn along the direction of the zipping movement. Then, the point of intersection between this line and the membrane zipping border is tracked for each consecutive still image or video frame. Using the scale as reference, the extracted pixel values are converted to the real distances $\mathrm{x}_{\text {meas }}$. As now the horizontal zipping distance is known along with the geometry of the actuator, the out-of-plane deflection of the membrane at the current zipping point $\mathrm{y}_{\text {meas }}$ can be calculated. For the simple geometry of a flat rigid electrode, this amounts to

$$
y_{\text {meas }}=x_{\text {meas }} \tan \beta
$$

with $\beta$ as the initial angle between the rigid electrode and the membrane. 


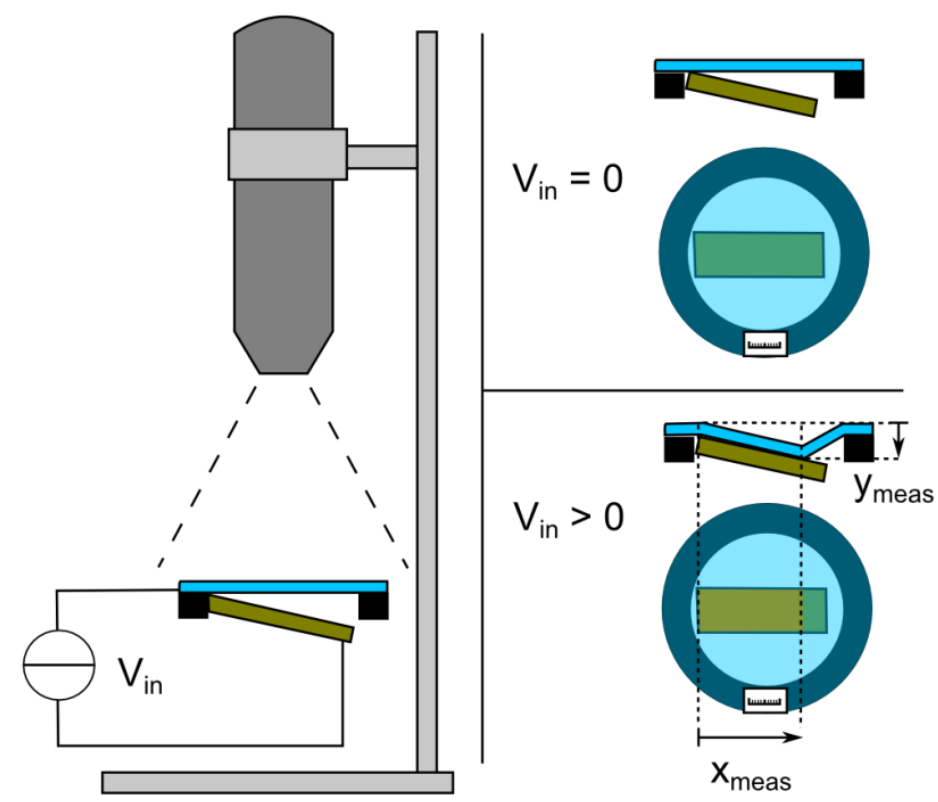

Figure 6: The visual test setup for the test actuators. Left: Side view of camera and specimen. Right: side view and top view of the actuator for both zero and nonzero voltage.
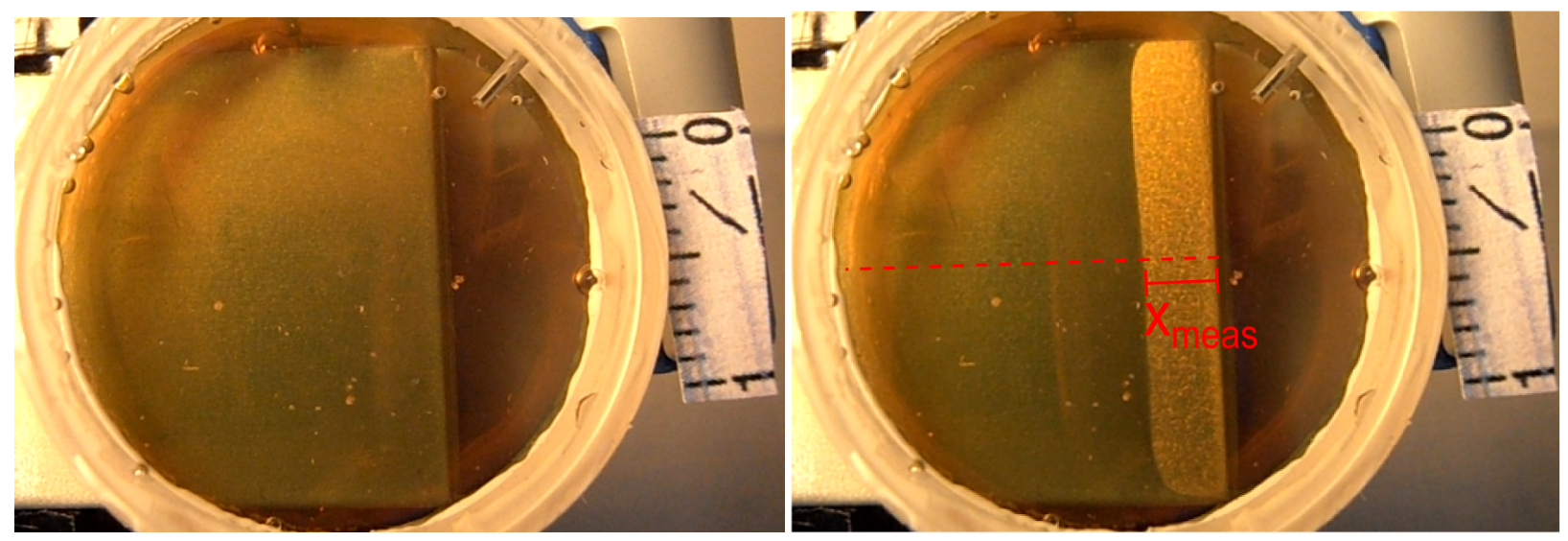

Figure 7: Sample images captured for the actuator characterization. Left: $V_{\text {in }}=0$. Right: $V_{\text {in }}>0$, showing zipping. The scale is in $\mathrm{cm}$.

\subsection{Static results}

The steady-state behavior of the actuators was quantified by sequentially applying constant voltages and taking still images of the resulting stable membrane deflection. Figure 8 shows the results for the case of the flat rigid electrode, and Figure 9 for the curved electrodes. In all static experiments, the voltage starts at $0 \mathrm{~V}$, goes up to a maximum voltage, go down to a minimal voltage and then up again to the maximum. This is done in order to see hysteresis effects.

In most devices, a certain minimal voltage is needed to start the zipping: e.g., for the flat rigid electrode in combination with a $0 \%$ prestretched membrane, $80 \mathrm{~V}$ is needed until any movement takes place. At that minimal voltage, however, the membrane snaps immediately to its corresponding deflection state. We will call this behavior the initial snap-in effect. After this has taken place, further increase of the voltage incrementally increases the deflection. When lowering the voltage again, the effect of the adhesion between the membrane and the insulator can be seen: depending on 
parameters such as implantation dose and prestretch, the membrane remains stuck to the surface. This introduces a hysteresis to the actuator behavior.

When the adhesion is especially high, such as in the case of the $25 \%$ prestretched membrane in Figure 8 , the voltage has to be reduced to less than half the maximal voltage until the membrane deflection decreases. The $50 \%$ prestretched membrane shows significantly less hysteresis, which is assumed to be due to the higher elastic forces. The membrane with $0 \%$ prestretch also shows little adhesion, which can be explained with the comparatively higher implantation dose (see Table 1).

In the case of the curved electrodes, some interesting general trends can be observed: The actuators with large electrode curvature radius show significantly higher maximal deflection than the ones with small radius. Also, they do not suffer from the initial snap-in effect: Continuous actuation is possible starting from little more than $0 \mathrm{~V}$. However, this benefit is associated with undesired adhesion: the small elastic restoring forces are not able to detach the membrane once it is deflected, causing non-reversible actuation.

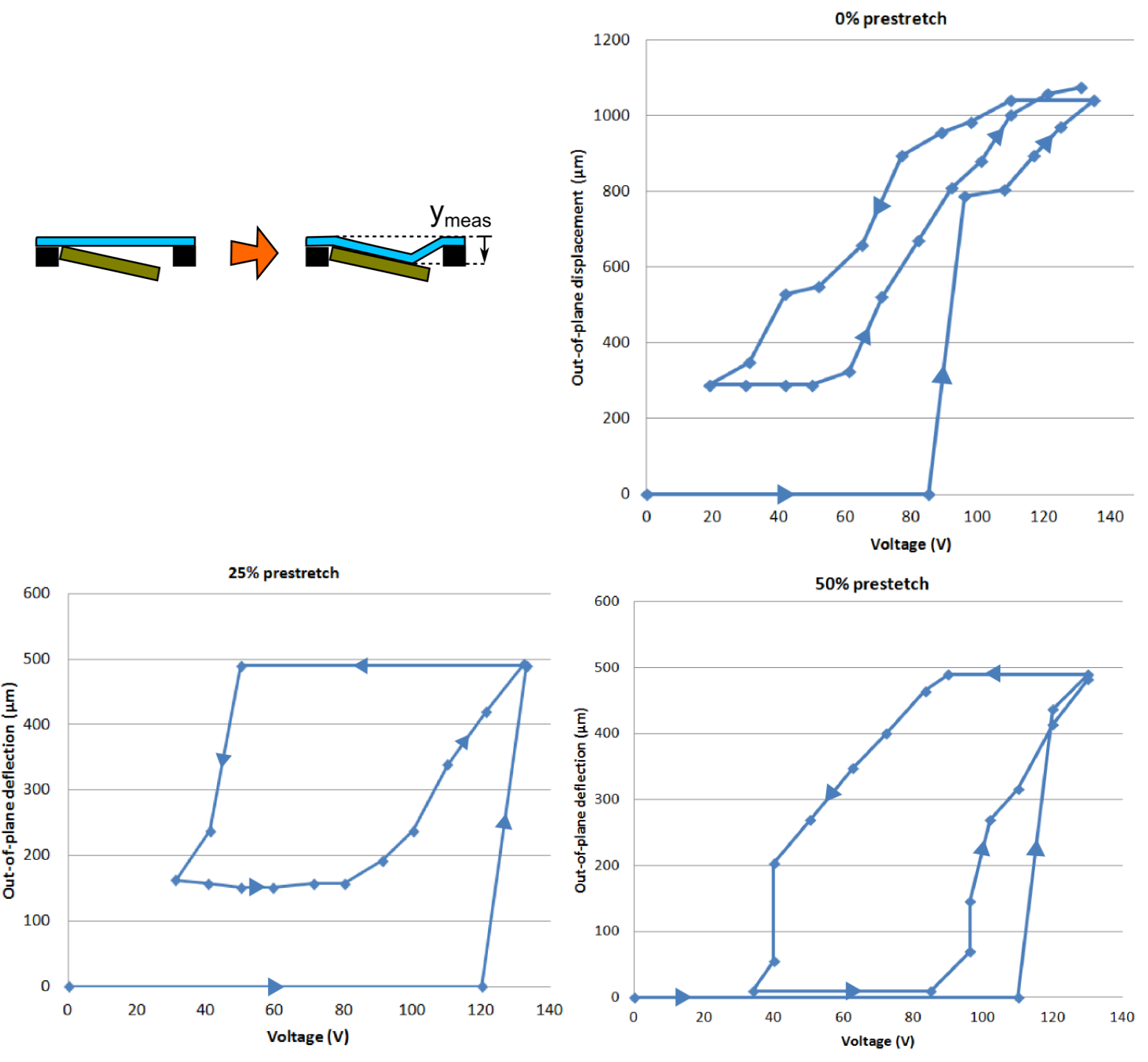

Figure 8: Experimental measurements of static behavior of actuators with flat electrodes for different membrane prestrains. The steady-state out-of-plane deflection ( $\mathrm{y}_{\text {meas }}$ ) is given as a function of applied voltage. Silicone membrane properties: Young's modulus $=0.4 \mathrm{MPa}$, thickness before prestretch $=50 \mu \mathrm{m}$. The rigid electrode has a width of $18 \mathrm{~mm}$ and is covered with a $15 \mu \mathrm{m}$ thick insulator layer. 

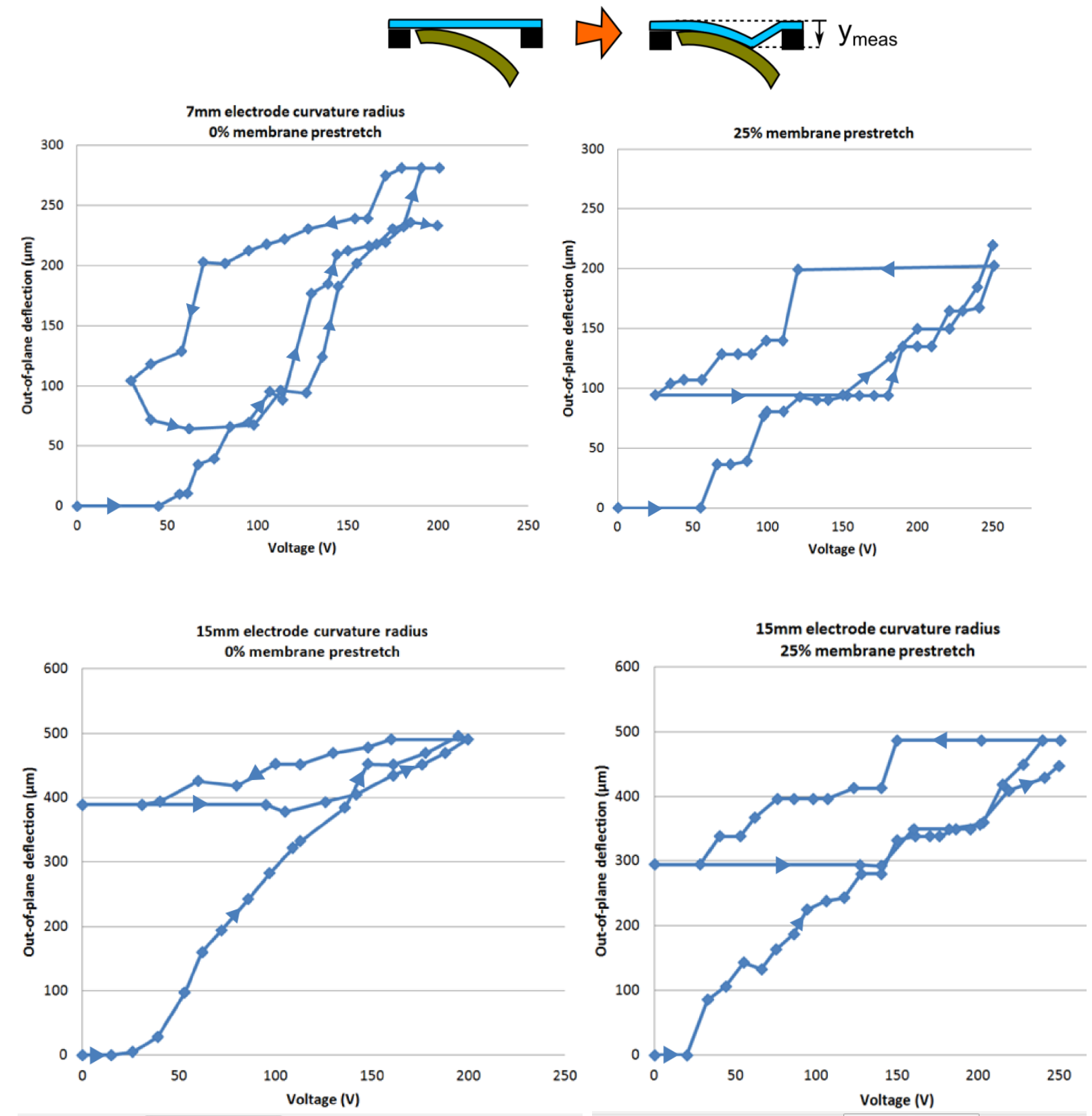

Figure 9: Static actuator behavior for curved electrodes. Silicone membrane properties: Young's modulus $=0.4 \mathrm{MPa}$, thickness before prestretch $=50 \mu \mathrm{m}$. The rigid electrode has a width of $8.5 \mathrm{~mm}$ and is covered with a $10 \mu \mathrm{m}$ thick insulator layer.

\subsection{Dynamic experiment results}

For the dynamic characterization, voltage steps of 0 to $160 \mathrm{~V}$ and 0 to $200 \mathrm{~V}$ were applied to the actuators. As described in section 2.2, video images were captured using a compact camera to measure the membrane's out-of-plane deflection $\left(\mathrm{y}_{\text {meas }}\right)$. Several experiments were conducted to evaluate the repeatability. Figure 11 shows the resulting step responses of a actuator containing liquid, Figure 11 of the devices without liquid. All measured actuators used the same rigid flat electrode in combination with different membranes. 
The response times the actuators need to go from $10 \%$ to $90 \%$ of their steady state deflection is shown in Table 3 . The sampling speed of the video acquisition system is $33 \mathrm{~ms}$, and the numerical values shown in the table are computed by linear interpolation between the sampling points. It should be noted that some of the computed values are in proximity to the sampling period, which limits the precision of those measurement results.

The actuators with $0 \%$ prestretch have two distinct time phases during their step response: A very quick rise up to a certain threshold followed by a slower movement until the final steady state value is reached. For instance, the actuators with $0 \%$ prestretch membranes without liquid reach $800 \mu \mathrm{m}$ deflection in less than $33 \mathrm{~ms}$. The steady state deflection of $1400 \mu \mathrm{m}$, however, is only reached after $600 \mathrm{~ms}$. The actuators involving liquid show a similar behavior. In contrast, the actuators with $25 \%$ and $50 \%$ prestretch move at a slower rate from the beginning of the step, and do not display these two time regimes.

A possible explanation of the initially fast movement of the $0 \%$ prestretched membrane is that it may be slightly sagging in its support ring. When the membrane is glued to the ring during fabrication, it should be perfectly flat. If for some reason this is not the case, the membrane will not be completely in plane with the ring surface, but will instead have some out-of-plane deflection caused by its own weight. When electrostatic forces pull on this membrane during zipping, its initial out-of-plane deflection will occur without the need of stretching the membrane: Until the membrane is brought to a minimal elongation, no tensile strain will occur within the membrane, and the elastomer material is accelerated without being held back by elastic forces. Then, as soon as the membrane starts to be actually stretched, a slower time regime would be expected, with time constants more close to those of prestrained membranes.

When comparing the prestretched membranes, it can be noted that the actuators with $50 \%$ prestretched membrane respond faster than the ones with only $25 \%$ prestretch. A surprising effect is the overshoot seen in the case of the $50 \%$ prestretched membranes: in the transient regime, up to $130 \%$ of the steady state deflection is reached. The same effect is visible in the case of the liquid-based membranes. Further investigation is ongoing to determine its cause.
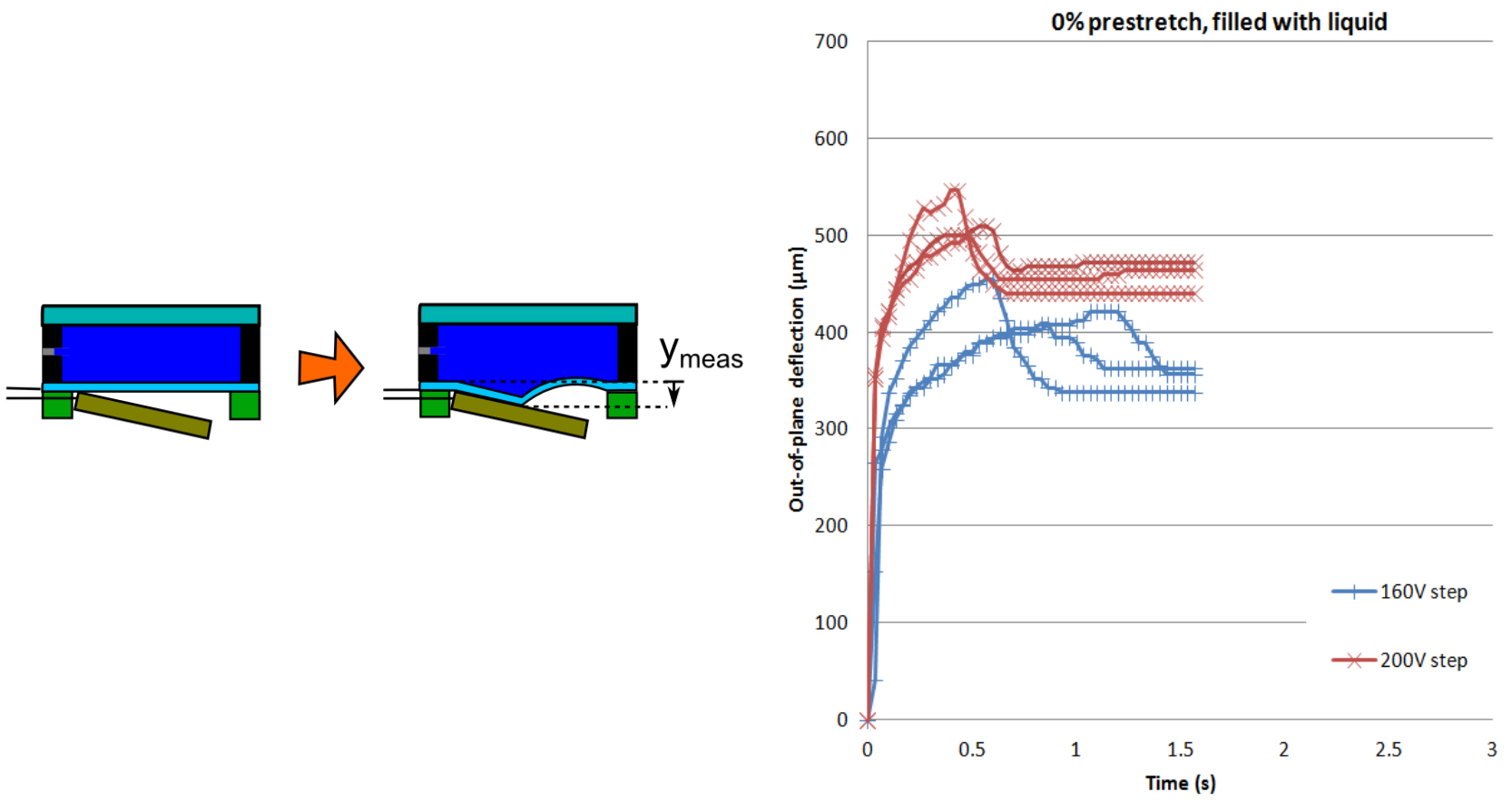

Figure 10: Step response of a zipping actuator filled with liquid, using the flat rigid electrode design. The time resolution of the measurements $\mathrm{T}_{\text {sample }}$ is $33 \mathrm{~ms}$. Silicone membrane properties: Young's modulus $=0.4 \mathrm{MPa}$, thickness before prestretch $=50 \mu \mathrm{m}$. The rigid electrode is covered with a $10 \mu \mathrm{m}$ thick insulator layer. 

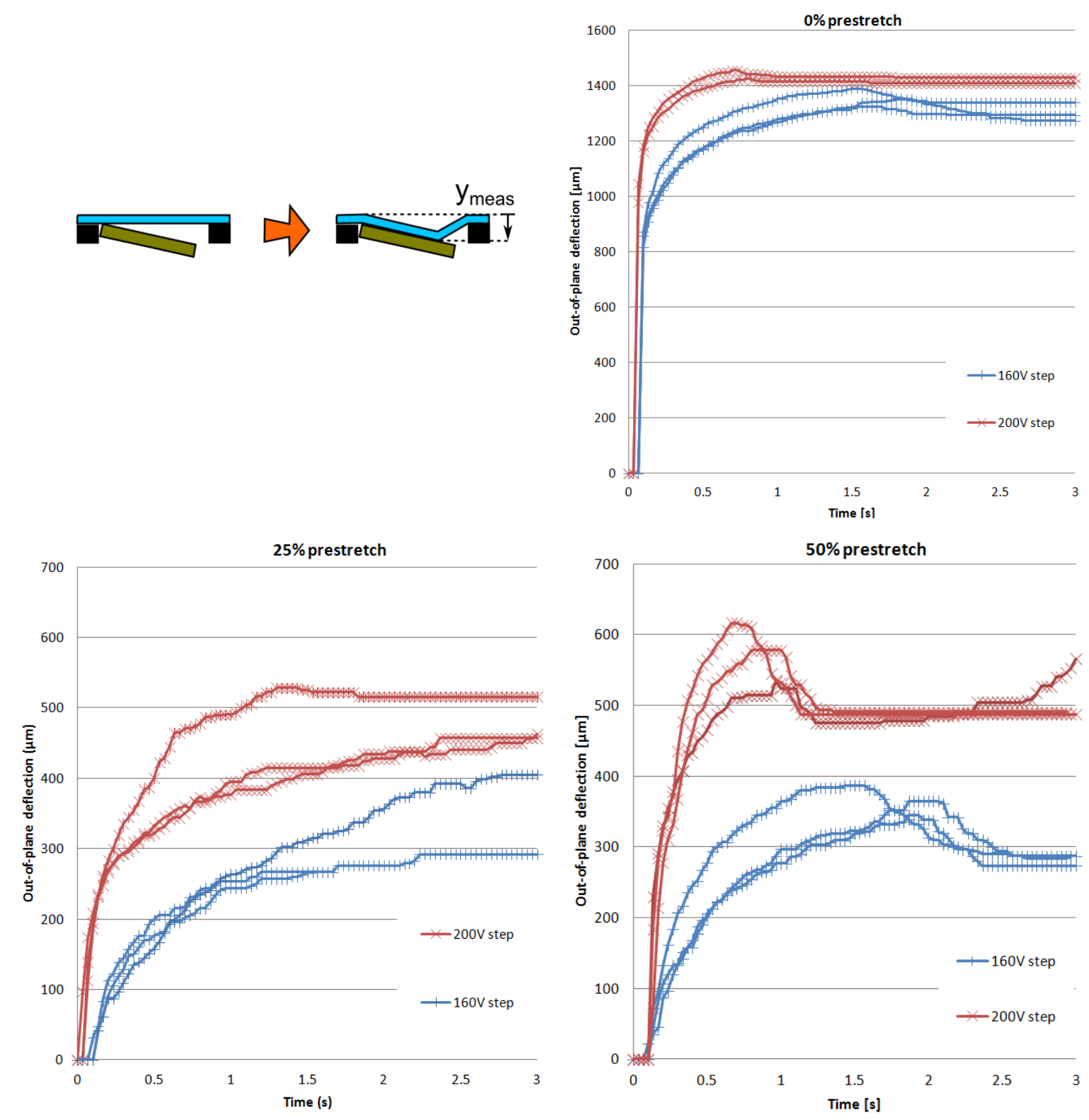

Figure 11: Step response of zipping actuators, using the flat rigid electrode design. The time resolution of the measurements $T_{\text {sample }}$ is $33 \mathrm{~ms}$. Silicone membrane properties: Young's modulus $=0.4 \mathrm{MPa}$, thickness before prestretch $=50 \mu \mathrm{m}$. In the case of the actuator shown left below, the membrane encloses a liquid. The rigid electrode is covered with a $10 \mu \mathrm{m}$ thick insulator layer. 
Table 3: Mean actuator rise time to go from $10 \%$ to $90 \%$ of the steady state deflection $\mathrm{y}_{\mathrm{ss}}$ resulting from the experiments shown in Figure 10 and Figure 11. N for each experiment is 2-3. The values are computed by linear interpolation between the sampling points. The sampling period is $33 \mathrm{~ms}$.

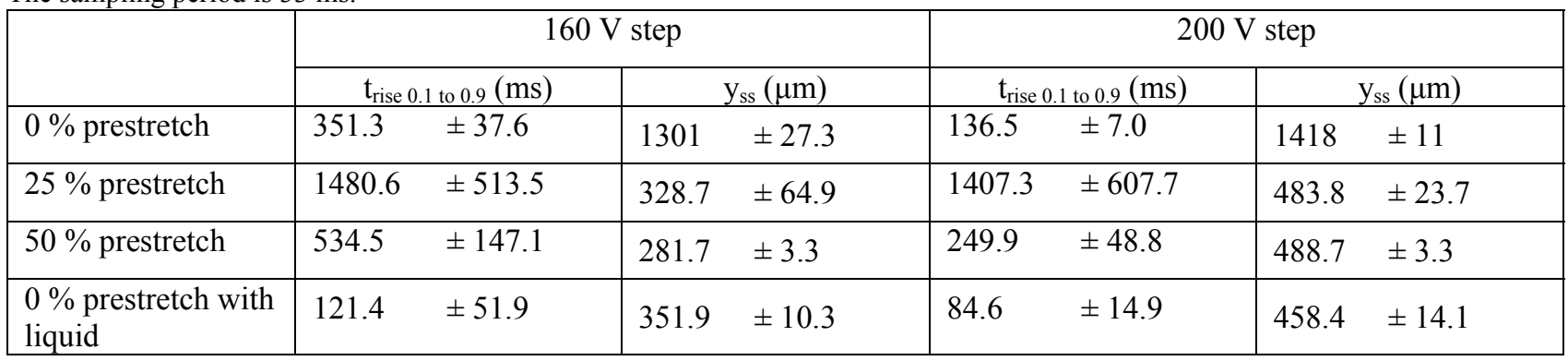

\section{CONCLUSIONS}

In this work we showed the feasibility of fabricating elastomer zipping actuators based on silicone membranes. Membrane deflection of up to $1400 \mu \mathrm{m}$ were reached at only $200 \mathrm{~V}$ actuation voltage. Membrane deflections of $800 \mu \mathrm{m}$ were reached in less than $35 \mathrm{~ms}$. For the first time, dynamic measurements of such zipping elastomer actuators were presented. The effect of the design parameters membrane prestretch and rigid electrode curvature was studied. We found that those variables have significant effects on actuator speed, maximal deflection and hysteresis.

\section{ACKNOWLEDGEMENTS}

We thank Optotune AG for their support for this work. This research was funded by CTI project 12342.2 PFNM-NM and by the Swiss National Science Foundation, grant \# 200020-130453.

\section{REFERENCES}

1. Pelrine, R., Kornbluh, R., Pei, Q. et al., "High-speed electrically actuated elastomers with strain greater than $100 \%$," Science, 287(5454), 836 (2000).

2. Bar-Cohen, Y., [Electroactive polymer (EAP) actuators as artificial muscles: reality, potential, and challenges] Society of Photo Optical, (2004).

3. Carpi, F., De Rossi, D., and Kornbluh, R., [Dielectric elastomers as electromechanical transducers: Fundamentals, materials, devices, models and applications of an emerging electroactive polymer technology] Elsevier Science, (2008).

4. Bell, D., Lu, T., Fleck, N. et al., "MEMS actuators and sensors: observations on their performance and selection for purpose," Journal of Micromechanics and Microengineering, 15, S153 (2005).

5. Grade, J. D., Jerman, H., and Kenny, T. W., "Design of large deflection electrostatic actuators,"

Microelectromechanical Systems, Journal of, 12(3), 335-343 (2003).

6. Yang, X., [A circular electrostatic zipping actuator for the application of a MEMS tunable capacitor] Massachusetts Institute of Technology, (2005).

7. Li, J., [Electrostatic zipping actuators and their applications to MEMS] Massachusetts Institute of Technology, (2004).

8. Brenner, M. P., Lang, J. H., Li, J. et al., "Optimum Design of an Electrostatic Zipper Actuator," NSTI-Nanotech. 2, 371-374 (2004).

9. Saif, M., Alaca, B., and Sehitoglu, H., "Analytical modeling of electrostatic membrane actuator for micro pumps," Journal of Microelectromechanical Systems, 8(3), 335-345 (1999).

10. van der Wijngaart, W., Ask, H., Enoksson, P. et al., "A high-stroke, high-pressure electrostatic actuator for valve applications," Sensors and Actuators A: Physical, 100(2-3), 264-271 (2002).

11. Sathe, A., Groll, E., and Garimella, S., "Analytical model for an electrostatically actuated miniature diaphragm compressor," Journal of Micromechanics and Microengineering, 18, 035010 (2008). 
12. Branebjerg, J., and Gravesen, P., "A new electrostatic actuator providing improved stroke length and force," Micro Electro Mechanical Systems. 6-11 (1992).

13. Horning, R., [PolyMEMS Actuator: A Polymer-Based Microelectromechanical (MEMS) Actuator with Macroscopic Action] Honeywell Inc. Plymouth MN, (2002).

14. Binh-Khiem, N., Matsumoto, K., and Shimoyama, I., "Polymer thin film deposited on liquid for varifocal encapsulated liquid lenses," Applied Physics Letters, 93(12), 124101-124101-3 (2008).

15. Rosset, S., [Metal Ion Implanted Electrodes for Dielectric Elastomer Actuators] École Polytechnique Fédérale de Lausanne, (2008).

16. Rosset, S., Niklaus, M., Dubois, P. et al., "Metal ion implantation for the fabrication of stretchable electrodes on elastomers," Advanced Functional Materials, 19(3), 470-478 (2009).

17. Niklaus, M., Rosset, S., Dadras, M. et al., "Microstructure of $5 \mathrm{keV}$ gold-implanted polydimethylsiloxane," Scripta Materialia, 59(8), 893-896 (2008).

18. Niklaus, M., [Metal-Ion Implanted Elastomers: Analysis of Microstructures and Characterization and Modeling of Electrical and Mechanical Properties] École Polytechnique Fédérale de Lausanne, (2010).

19. Dubois, P., Rosset, S., Niklaus, M. et al., "Metal Ion Implanted Compliant Electrodes in Dielectric Electroactive Polymer (EAP) Membranes," Advances in Science and Technology, 61, 18-25 (2009).

20. Creton, C., and Leibler, L., "How does tack depend on time of contact and contact pressure?," Journal of Polymer Science Part B: Polymer Physics, 34(3), 545-554 (1996).

21. McPherson, J. W., Kim, J., Shanware, A. et al., "Trends in the ultimate breakdown strength of high dielectricconstant materials," Electron Devices, IEEE Transactions on, 50(8), 1771-1778 (2003). 